\title{
Mn-doped InAs self-organized diluted magnetic quantum-dot layers with Curie temperatures above $300 \mathrm{~K}$
}

\author{
M. Holub, S. Chakrabarti, S. Fathpour, and P. Bhattacharyaa) \\ Department of Electrical Engineering and Computer Science, Solid State Electronics Laboratory, \\ University of Michigan, Ann Arbor, Michigan 48109-2122 \\ Y. Lei \\ Electron Microscopy Center, Material Science Division, Argonne National Laboratory, \\ Argonne, Illinois 60439 \\ S. Ghosh \\ Department of Electrical and Computer Engineering, University of Illinois at Chicago, \\ Chicago, Illinois 60607
}

(Received 4 February 2004; accepted 14 June 2004)

\begin{abstract}
The magnetic and structural properties of InAs:Mn self-organized diluted magnetic quantum dots grown by low-temperature $\left(\sim 270^{\circ} \mathrm{C}\right)$, solid-source molecular-beam epitaxy using a very low InAs growth rate $(<0.1 \mathrm{ML} / \mathrm{s})$ are investigated. A Curie temperature $\left(T_{C}\right)$ of $\sim 350 \mathrm{~K}$ is measured in a sample grown with a $\mathrm{Mn} / \mathrm{In}$ flux ratio of 0.15 . Electron energy-loss spectroscopy confirms that most of the Mn remains within the InAs quantum dots. We propose as a possible explanation for this high $T_{C}$ the effects of magnetic and structural disorder introduced by a random incorporation and inhomogeneous distribution of $\mathrm{Mn}$ atoms amongst the InAs quantum dots. () 2004 American Institute of Physics. [DOI: 10.1063/1.1781361]
\end{abstract}

Among III-V diluted magnetic semiconductors (DMSs), $(\mathrm{Ga}, \mathrm{Mn}) \mathrm{As}$ and its heterostructures have elicited the greatest amount of interest for their potential application in semiconductor spintronic devices. Recent investigations have shown that the ferromagnetic transition, or Curie, temperature $\left(T_{C}\right)$ may be raised to $150 \mathrm{~K}$ in thin $(15 \mathrm{~nm})(\mathrm{Ga}, \mathrm{Mn}) \mathrm{As}$ epilayers, ${ }^{1} 160 \mathrm{~K}$ in $(\mathrm{Ga}, \mathrm{Mn})$ As trilayer structures, ${ }^{2}$ and $172 \mathrm{~K}$ in $\mathrm{Mn} \delta$-doped GaAs/p-AlGaAs triangular quantum wells ${ }^{3}$ after low-temperature annealing. For electronic and optoelectronic spin injection devices, it is clearly desirable to achieve spin injection and device operation at room temperature, rendering $(\mathrm{Ga}, \mathrm{Mn}) \mathrm{As}$ unsuitable for such applications at the present time.

In this letter, we report the growth of Mn-doped InAs self-organized quantum dots, or "diluted magnetic quantum dots" (DMQDs). The magnetic properties of the quantum-dot layers have been investigated by superconducting quantum interference device (SQUID) magnetometry. Atomic force microscopy (AFM), x-ray energy dispersive spectroscopy (XEDS), electron energy-loss spectroscopy (EELS), and transmission electron microscopy (TEM) were used to examine the microstructure of these materials. A possible explanation for the high $T_{C}$ observed is offered based on recent theoretical studies ${ }^{4-7}$ of hole-mediated ferromagnetism, accounting for the effect of disorder.

Several InAs:Mn quantum-dot multilayers of varying $\mathrm{Mn}$ concentration were grown by low-temperature molecular-beam epitaxy (LT-MBE) on semi-insulating (100)oriented GaAs substrates in a Varian GEN-II chamber. ${ }^{8}$ All samples were grown using a relatively low $\mathrm{As}_{4}$ : $\mathrm{Ga}$ beam equivalent pressure (BEP) ratio $(\sim 16: 1)$, a very low InAs growth rate $(\sim 0.07 \mathrm{ML} / \mathrm{s})$, and a growth temperature in the range of $250-280^{\circ} \mathrm{C}$. A radiatively coupled thermocouple is

a)Electronic mail: pkb@eecs.umich.edu used to monitor the temperature of the substrate, which is In mounted to a molybdenum block during growth. After depositing a $500-\mathrm{nm} \mathrm{GaAs}$ buffer layer at $610^{\circ} \mathrm{C}$, the substrate was cooled to a temperature in the range of $250-280^{\circ} \mathrm{C}$ for the growth of a 100-nm LT-GaAs buffer layer followed by the InAs:Mn QD multilayers. After deposition of a 2.0-ML InAs wetting layer, the Mn effusion cell shutter was opened for the growth of 2.6 ML of InAs:Mn QDs, upon which the RHEED pattern become dim and then spotty. A 45-s growth interruption was allowed for dot formation before capping with $50-56 \mathrm{~nm}$ of undoped GaAs. Finally, a 30-nm GaAs capping layer was deposited following the growth of ten InAs:Mn QD layers. The heterostructure for a representative sample is shown in Fig. 1.

Initial optimization of the dot growth conditions was accomplished via AFM. The three-dimensional (3D) AFM image for 4.7 ML of InAs:Mn deposited on LT-GaAs using a very low InAs growth rate $(0.05 \mathrm{ML} / \mathrm{s})$, a substrate temperature of $280^{\circ} \mathrm{C}$, and a $\mathrm{Mn} / \mathrm{In}$ flux ratio of 0.05 , indicates that the dots are well formed. The base length and height of the dots are $35-37$ and $13-15 \mathrm{~nm}$, respectively; the dot density

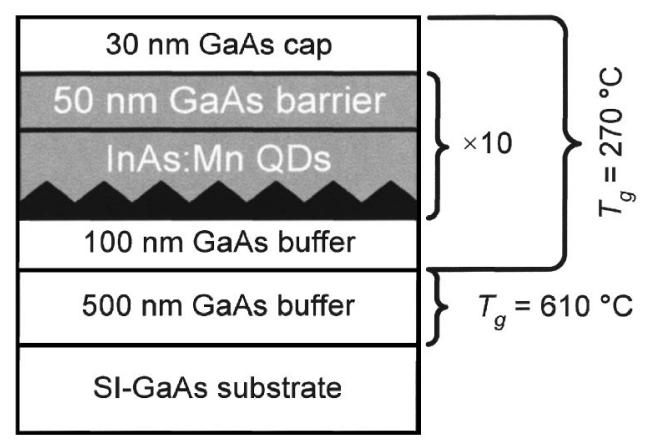

FIG. 1. Schematic heterostructure of a typical InAs:Mn quantum-dot multilayer sample grown by LT-MBE. 

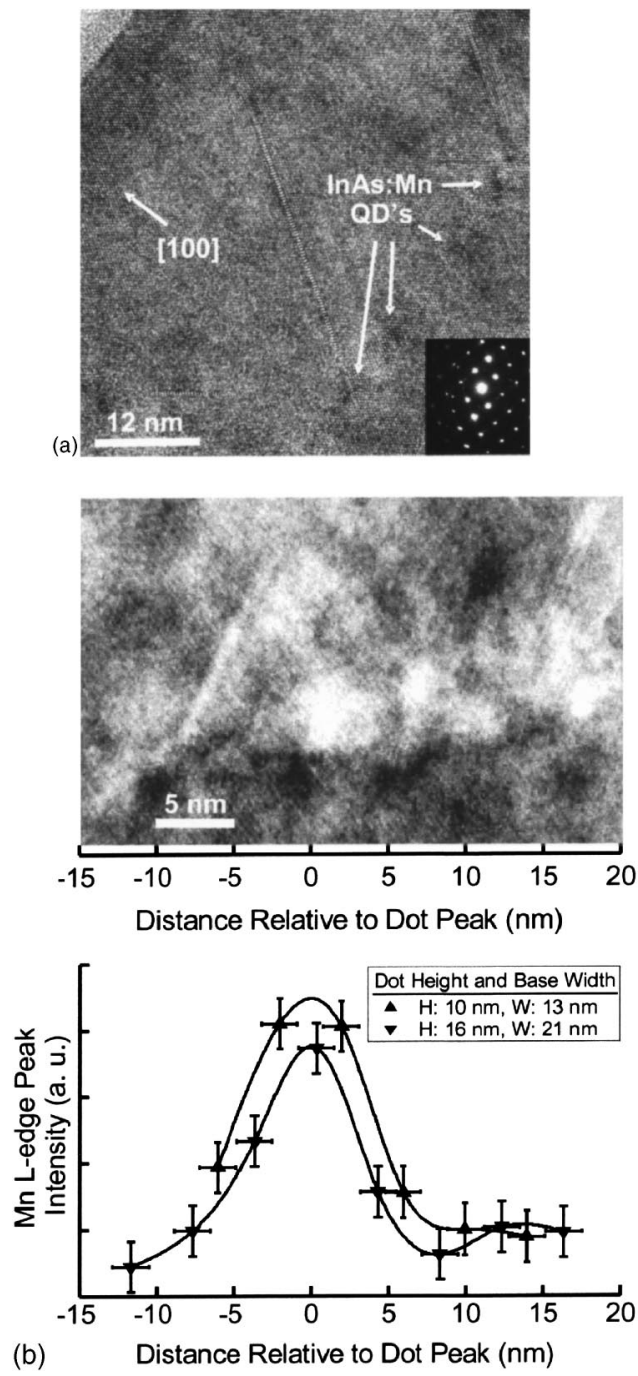

FIG. 2. (a) High-magnification TEM bright field image of an InAs:Mn quantum-dot layer taken with $\langle 011\rangle$ projection. A representative electrondiffraction pattern of the GaAs matrix surrounding an InAs:Mn QD layer is shown in the inset. (b) High-resolution TEM image of an InAs:Mn quantum dot (top). In-plane EELS profile (2-nm lateral resolution) showing the distribution of $\mathrm{Mn}$ at a single InAs:Mn quantum dot as represented by the Mn $L$-edge peak intensity vs position (bottom).

is approximately $3 \times 10^{9} \mathrm{~cm}^{-2}$. Thus, InAs:Mn QDs grown by LT-MBE are almost twice as large as self-organized InAs QDs grown at a substrate temperature of $500{ }^{\circ} \mathrm{C}$. Undoped InAs QDs were grown by low-temperature epitaxy in order to investigate the effect of Mn on dot formation. The AFM images of the resultant dots exhibit smaller dot size and higher dot density, suggesting that Mn plays the role of a surfactant, ${ }^{9,10}$ alters the surface kinetics, and enhances the adatom migration rates to result in larger dots and a smaller dot density in the presence of $\mathrm{Mn}$.

Five layers of InAs:Mn QDs were grown by LT-MBE for structural characterization, using a procedure similar to that described above with 50-nm GaAs buffer layers and a substrate temperature of $280^{\circ} \mathrm{C}$. TEM bright field imaging and diffraction analysis [Fig. 2(a)] show that the InAs:Mn multilayer structure is fully pseudomorphic with respect to the GaAs substrate, but some dislocations, stacking faults, and point defects are generated during low-temperature epitaxy. Neither evidence of hexagonal, NiAs-type MnAs clusters was observed by TEM imaging nor was a second lattice structure detected by diffraction analysis. A high-resolution, cross-sectional TEM image of a single dot [Fig. 2(b)] shows that InAs:Mn QDs grown by LT-MBE maintain a zincblende crystal structure and are near pyramidal in shape, having a height and base width of 12 and $21 \mathrm{~nm}$, respectively. XEDS analysis confirms the presence of $\mathrm{Mn}$ in each of the five QD layers and finds the average $\mathrm{Mn} / \mathrm{In}$ ratio over these layers to be 0.236 .

Nanoscale variation of the Mn composition within a single QD layer is resolved by EELS analysis using an inplane Mn $L$-edge profile; the results are shown in Fig. 2(b). We find that the majority of the Mn dopants are contained within the dot, as indicated by an increase in the $\mathrm{Mn} L$-edge peak intensity when the EELS probe scanned over an InAs:Mn dot, but that a portion of the Mn atoms have diffused outside of the dots into the surrounding GaAs matrix. This result corroborates the work of Ofuchi et al., ${ }^{11}$ wherein fluorescence extended $\mathrm{x}$-ray absorption fine-structure analysis revealed that the majority of the Mn atoms incorporated substitutionally on In lattice sites of similarly grown (In, $\mathrm{Mn})$ As QDs. Furthermore, we note from comparison of the $M n L$-edge profile peak intensities obtained from two independent EELS scans within a single InAs:Mn QD layer that the Mn concentration varies perceptibly from dot to dot. This observation suggests that significant positional disorder of $\mathrm{Mn}$ atoms is present in our samples as a result of size fluctuations inherent in self-assembled InAs QDs.

Evaluation of the magnetic properties for (In,Mn)As QDs has previously been hampered by the relatively small number of total $\mathrm{Mn}$ atoms. However, we circumvent this problem through the growth of multiple InAs:Mn QD layers having a relatively high $\mathrm{Mn}$ concentration, which are separated by undoped GaAs buffers of sufficient thickness to allow for surface recovery. The magnetic properties of the InAs:Mn QD multilayer samples were studied using a Quantum Design MPMS-5 SQUID magnetometer. Measurement of the magnetic moment versus magnetic field reveals a clear hysteresis loop, indicating ferromagnetic order in the InAs:Mn QD multilayer samples. The field dependence of the magnetic moment per unit sample area obtained at various temperatures is shown in Fig. 3(a). Hysteresis is observed when the field is applied either parallel or perpendicular to the sample surface, as seen in the inset of Fig. 3(b), but is less pronounced for the case of a perpendicularly applied field. This result suggests that magnetic anisotropy favors in-plane magnetization - in contrast to previous reports on InAs:Mn QDs ${ }^{12}$ - as expected for compressively strained InAs:Mn QDs grown atop a LT-GaAs buffer layer.

After magnetizing the zero-field-cooled sample at $4.2 \mathrm{~K}$ for $60 \mathrm{~s}$ in the presence of $1 \mathrm{~T}$, the temperature dependence of the magnetic moment was measured upon heating with a 50-Oe field applied either parallel or perpendicular to the sample surface. For convenience, the temperature at which the magnetic moment vanishes under a small magnetic field is taken as the Curie temperature $T_{C}$, which, as seen in Fig. $3(\mathrm{~b})$, is as high as $350 \mathrm{~K}$ in a sample grown using a $\mathrm{Mn} / \mathrm{In}$ flux ratio of 0.15 . The reported Curie temperatures of $\mathrm{MnAs}$ and $\mathrm{MnGa}$ clusters in $\mathrm{Ga}(\mathrm{Mn}) \mathrm{As}$ are $318 \mathrm{~K}$ and above $400 \mathrm{~K},{ }^{13}$ respectively. Thus, a Curie temperature of $350 \mathrm{~K}$ suggests a magnetic response that is either unique to InAs:Mn QDs or MnAs-rich MnGaAs clusters, though significant clustering is not indicated by our material characterization as discussed above. 

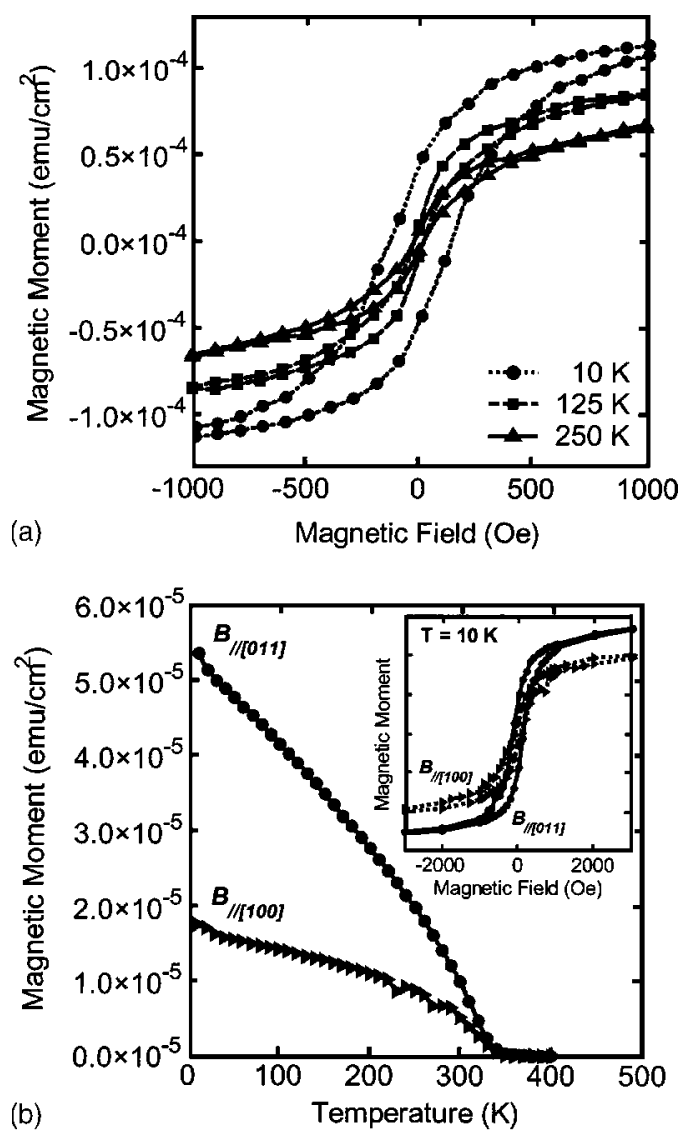

FIG. 3. (a) Hysteresis loops for a ten-layer InAs:Mn QD sample measured by SQUID magnetometry at $T=10,125$, and $250 \mathrm{~K}$; (b) magnetic moment per unit area vs temperature for a ten-layer InAs:Mn QD sample measured by SQUID magnetometry in the presence of a 50-Oe field. The inset shows the hysteresis loops for the same sample with in-plane and out-of-plane magnetization obtained at $10 \mathrm{~K}$. A temperature-independent, diamagnetic contribution from the GaAs substrate is subtracted from the measured data.

The observed Curie temperatures in the InAs:Mn QD multilayer samples are significantly higher than those observed in either $(\mathrm{Ga}, \mathrm{Mn})$ As or $(\mathrm{In}, \mathrm{Mn})$ As thin films grown by LT-MBE. It is now well established that ferromagnetism in bulk DMSs results when delocalized or weakly localized holes can mediate $p$ - $d$ exchange coupling between themselves and the localized spins of impurity Mn atoms. While theoretical models neglecting the effect of disorder on magnetic properties and treating $p-d$ exchange using a mean-field approximation provide a Curie temperature in reasonable agreement with experimental findings, ${ }^{14}$ ferromagnetism in quantum dots is more complex as the effects of epitaxial strain, quantum confinement, and $\operatorname{disorder}^{4-7}$ are expected to play a key role in determining their magnetic properties. We note, in particular, that the degree of disorder in DMQDs is larger than in bulk, due not only to substitutional disorder but also to inhomogeneous $\mathrm{Mn}$ incorporation among neighboring DMQDs and irregularities in dot size and distribution unavoidably introduced during self-assembly. As shown by Berciu and Bhatt, ${ }^{4}$ when Mn positional disorder is accounted for in a mean-field description of ferromagnetism in lowdoping DMSs, the Curie temperature can increase signifi- cantly with the degree of disorder, as holes migrate toward regions of higher Mn concentration to lower their total energy, thereby stabilizing a ferromagnetic state at higher temperatures than in a uniform system. Theoretical studies by Bouzerar et al., ${ }^{5}$ accounting for a disordered carrier distribution within a nonperturbative Ruderman-Kittel-KasuyaYosida (RKKY) treatment, similarly predict higher Curie temperatures in DMSs than are suggested by standard RKKY calculations. Secondly, the presence of strong charge and spin disorder make carrier distribution and localization relevant issues in DMQDs. Hole localization by the trapping potential of Mn impurities can create a spin polarization of $\mathrm{Mn}$ atoms within the span of its wave function to form bound magnetic polarons, ${ }^{15}$ the interaction of which characterizes the ferromagnetic behavior of insulating, doped DMSs. ${ }^{16}$ Carrier confinement within a QD is expected to strengthen hole localization and subsequently enhance the thermal stability of magnetic polarons, which may explain ferromagnetism in DMQDs at high temperatures.

This work is being supported by the Office of Naval Research under Grant No. N00014-02-1-0899 and the Army Research Office (MURI program) under Grant No. DAAD19-99-1-0198. Electron microscopy was carried out in the Electron Microscopy Center at Argonne National Laboratory, which is supported by the U.S. Department of Energy, Office of Science, under Contract No. W-31-109-ENG-38. M.H. acknowledges support under a National Science Foundation Graduate Research Fellowship.

${ }^{1}$ K. C. Ku, S. J. Potashnik, R. F. Wang, S. H. Chun, P. Schiffer, N. Samarth, M. J. Seong, A. Mascarenhas, E. Johnston-Halperin, R. C. Myers, A. C. Gossard, and D. D. Awschalom, Appl. Phys. Lett. 82, 2302 (2003).

${ }^{2}$ D. Chiba, K. Takamura, F. Matsukura, and H. Ohno, Appl. Phys. Lett. 82, 3020 (2003).

${ }^{3}$ A. M. Nazmul, S. Sugahara, and M. Tanaka, Phys. Rev. B 67, 241308 (2003).

${ }^{4}$ M. Berciu and R. N. Bhatt, Phys. Rev. B 69, 045202 (2004).

${ }^{5}$ G. Bouzerar, J. Kudrnovský, and P. Bruno, Phys. Rev. B 68, 205311 (2003).

${ }^{6}$ A. L. Chudnovskiy and D. Pfannkuche, Phys. Rev. B 65, 165216 (2002).

${ }^{7}$ M. P. Kennett, M. Berciu, and R. N. Bhatt, Phys. Rev. B 66, 045207 (2002).

${ }^{8}$ P. Bhattacharya, S. Fathpour, S. Chakrabarti, M. Holub, and S. Ghosh, Proceedings of the Materials Research Society Symposium, Vol. 794, edited by A. G. Norman, R. S. Goldman, R. Noetzel, and G. B. Stringfellow (Materials Research Society, Warrendale, PA, 2003), pp. 215-225.

${ }^{9}$ S. P. Guo, A. Shen, F. Matsukura, Y. Ohno, and H. Ohno, J. Cryst. Growth 201-202, 684 (1999).

${ }^{10}$ S. P. Guo, A. Shen, H. Yasuda, Y. Ohno, F. Matsukura, and H. Ohno, J. Cryst. Growth 208, 799 (2000).

${ }^{11}$ H. Ofuchi, T. Kubo, M. Tabuchi, Y. Takeda, F. Matsukura, S. P. Guo, A. Shen, and H. Ohno, J. Appl. Phys. 89, 66 (2001).

${ }^{12}$ Y. K. Zhou, H. Asahi, J. Asakura, S. Okumura, K. Asami, and S. Gonda, J. Cryst. Growth 221, 605 (2000).

${ }^{13}$ J. Shi, J. M. Kikkawa, R. Proksch, T. Schaeffer, D. D. Awschalom, G. Medeiros-Ribeiro, and P. M. Petroff, Nature (London) 377, 707 (1995); J. Shi, J. M. Kikkawa, D. D. Awschalom, G. Medeiros-Ribeiro, P. M. Petroff, and K. Babcock, J. Appl. Phys. 79, 5296 (1996).

${ }^{14}$ T. Dietl, H. Ohno, F. Matsukura, J. Cibert, and D. Ferrand, Science 287, 1019 (2000).

${ }^{15}$ T. Dietl and J. Spałek, Phys. Rev. B 28, 1548 (1983).

${ }^{16}$ A. C. Durst, R. N. Bhatt, and P. A. Wolff, Phys. Rev. B 65, 235205 (2002). 